\title{
Case finding for COPD in primary care: a qualitative study of the views of health professionals
}

\author{
This article was published in the following Dove Press journal: \\ International Journal of COPD \\ 28 August 2015 \\ Number of times this article has been viewed
}

\section{Shamil Haroon \\ Rachel E Jordan \\ David A Fitzmaurice \\ Peymane Adab}

School of Health and Population Sciences, University of Birmingham, Edgbaston, Birmingham, UK

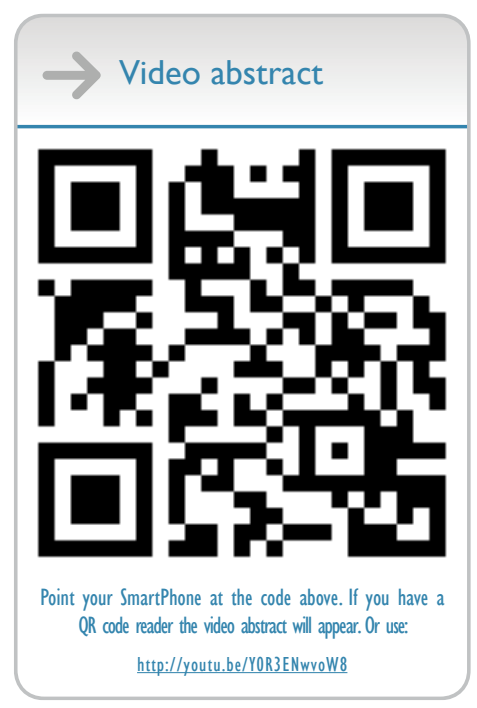

Correspondence: Peymane Adab; Rachel E Jordan

School of Health and Population Sciences, University of Birmingham, Edgbaston, Birmingham BI5 2TT, UK

Tel +44 I2 I 4 I 43777

Email p.adab@bham.ac.uk;

r.e.jordan@bham.ac.uk
Background: Chronic obstructive pulmonary disease (COPD) is common but largely underdiagnosed. Case-finding initiatives have been evaluated in primary care, but few studies have explored the views of service providers on implementing them in practice.

Aim: To explore the views of primary health care providers on case finding for COPD.

Methods: A total of 20 semi-structured interviews were conducted from March 2014 to September 2014 among general practitioners, nurses, and managers from practices participating in a large COPD case-finding trial based in primary care in the West Midlands, UK. Participants' views were sought to explore perceived benefits, harms, barriers, and facilitators to implementing COPD case finding in practice. Interviews were transcribed and analyzed using the framework method.

Results: Participants felt that case finding improves patient care but also acknowledged potential harms to providers (increase in workload) and to patients (overdiagnosis). Insufficient resources, poor knowledge of COPD, and limited access to diagnostic services were viewed as barriers to diagnosis, while provision of community respiratory services, including COPD specialist nurses, and support from secondary care were thought to be facilitators. Participants also expressed a need for more education on COPD for both patients and clinicians.

Conclusion: Care providers believe that early detection of COPD improves patient care but also has accompanying harms. Barriers to diagnosing COPD, such as insufficient expertise in primary care and limited access to diagnostic services in the community, should be explored and addressed. The knowledge and attitudes of the public about COPD and its symptoms should also be investigated to inform future education and awareness-raising strategies.

Keywords: chronic obstructive pulmonary disease, primary care, diagnosis, qualitative research

\section{Introduction}

Chronic obstructive pulmonary disease (COPD) is a major cause of morbidity and mortality ${ }^{1}$ and represents a significant cost to health services and society. ${ }^{2}$ However, much of the disease burden remains undiagnosed, ${ }^{3}$ and there has been a policy drive to identify COPD early through systematic case finding. ${ }^{4}$ This has been accompanied by the evaluation of a number of case-finding strategies. ${ }^{5,6}$ However, there has been a paucity of research exploring the views of primary care practitioners on these initiatives or factors influencing the ability of health services to screen for and diagnose COPD.

A study in Tasmania conducted semi-structured interviews and focus groups to explore the views of patients with COPD and their general practitioners (GPs) on factors influencing the diagnosis of COPD. ${ }^{7}$ This study found that GPs intentionally 
avoided early diagnosis as a result of harboring nihilistic attitudes toward COPD and misperceiving patient expectations. Patients reported receiving the diagnosis from other sources and were frustrated by delayed diagnosis.

Another study by the same authors randomly assigned eight practices to deliver either optimized usual care or opportunistic assessment with spirometry for ever smokers aged over 35 years who routinely attend primary care. ${ }^{8}$ At the end of the study, focus groups were conducted with participating GPs to explore their views on each approach. They felt that organized follow-up, especially with spirometry, was essential but would increase an already high workload and increase costs for patients. They also expressed a need for assistance with interpreting spirometry but felt its use prompted them to record their patients' smoking status and initiate discussions about smoking cessation. Some also questioned the value of diagnosing COPD in the absence of a cure.

Since these studies were published, much has been done nationally and internationally to emphasize the importance of undiagnosed $\mathrm{COPD}^{9}$ and provide education and guidance on its diagnosis and management. ${ }^{4,10}$ For example in the UK, the Quality and Outcomes Framework, which forms part of the reimbursement system for primary care, includes a number of quality indicators for the diagnosis and management of COPD, such as the recording of spirometry results for all new diagnoses. ${ }^{11}$ However, it is unclear whether attitudes toward the diagnosis of COPD among health professionals have changed with the introduction of these policies. In all, 54 general practices were recently enrolled in a large pragmatic cluster randomized controlled trial in the West Midlands, UK, comparing the effectiveness and cost-effectiveness of targeted case finding for COPD against routine care. ${ }^{12}$ Interviews were undertaken with participating health care providers to gain insights into their views on case finding for COPD and to discern factors that might influence their ability to make a diagnosis.

\section{Method Study design}

Semi-structured interviews were undertaken with primary care service providers by the lead investigator ( $\mathrm{SH}$ ). Interviews were conducted from March 2014 to September 2014 and were audio recorded and transcribed verbatim. Memos were made shortly after each interview to summarize key points and reflections.

\section{Participants}

One GP, nurse, and manager were invited from each of the 54 general practices participating in the TargetCOPD trial. ${ }^{12}$ Practices were selected to represent a wide range of population and practice characteristics. Eligible participants were posted an invitation letter as well as up to two reminders.

\section{Sample size}

We aimed to recruit five to ten participants of each profession across at least five general practices with a minimum sample size of 20. Eventual sample size was determined by the reaching of theoretical saturation (ie, no new concepts arising from the data). ${ }^{13,14}$

\section{Interviews}

One-to-one semi-structured interviews were conducted either at practices $(n=9)$ or over the telephone $(n=11)$ using a topic prompt (although questions could be asked outside the topic prompt if felt appropriate; Table 1) and had a mean duration of 23 minutes (range: 13-38 minutes). Repeat interviews were not conducted, and transcripts were not returned to participants for comment.

\section{Analysis}

Interviews were analyzed using the framework method. ${ }^{15}$ In brief, transcripts were read to identify codes or themes referring to specific topics. Two transcripts considered to be particularly rich and informative were independently coded by three of the

Table I Topic prompt

- Please tell me about any experience you have had looking after patients with COPD.

- What are your thoughts on screening or case finding for COPD?

- How do you think it would be best to identify undiagnosed patients with COPD in the community?

- Does your practice take part in any COPD case-finding activities? Please tell me about this.

- What might be the barriers to case finding and identifying patients with COPD?

- What would help primary care services identify patients with COPD?

- We are developing an electronic tool for GPs that will help them identify which of their patients are at high risk of undiagnosed COPD. Do you think such a tool would be useful? Do you think it would be used in practice, and if so, in what way?

- Is there anything else you would like to comment about screening or case finding for COPD?

Abbreviation: COPD, chronic obstructive pulmonary disease. 
authors (SH, REJ, and PA) and compared to create an initial coding framework. Coding of all subsequent transcripts was performed by the lead investigator and built on this framework. A framework matrix was then constructed, tabulating quotes by their associated codes and participant type. Emergent themes were then discussed and finalized by three authors. The analysis was performed using NVivo version 10 .

\section{Ethical approval}

Ethical approval was provided by the Solihull National Research Ethics Service committee (reference: 11/WM/ 0403). All identifiable data were held on an encrypted database.

\section{Results}

\section{Practice and participant characteristics}

A total of 162 care providers were invited to participate, of which 20 participants (ten GPs, seven practice nurses, and three practice managers) from 16 practices were interviewed (Tables 2 and 3). Practices had a range of patient list sizes with most having 5-10,000 patients and the majority serving relatively socioeconomically deprived populations. Approximately one-third of practices had been in the case-finding arm of the trial, and all practices had been involved in recruiting patients for a large COPD cohort study (the Birmingham COPD Study). Most GPs (70\%) interviewed were male, and all practice nurses and managers were female. Participants had been in practice on average for 13 or more years.

\section{Views on case finding}

Participants were generally of the opinion that early detection of COPD was beneficial for both patients and health services. Several participants felt that early detection improved smoking cessation, helped instigate positive changes to other

Table 2 Practice characteristics

\begin{tabular}{llll}
\hline Characteristics of included practices & $\mathbf{N}$ & $\mathbf{( \% )}$ \\
\hline Total number of practices & & 16 & $(100)$ \\
Patient list size & $0-5,000$ & 5 & $(31.3)$ \\
& $5,000-10,000$ & 8 & $(50.0)$ \\
& $>10,000$ & 3 & $(18.8)$ \\
IMD quintile & I (most deprived) & 8 & $(50.0)$ \\
& 2 & 1 & $(6.3)$ \\
& 3 & 3 & $(18.8)$ \\
& 4 & 4 & $(25.0)$ \\
Intervention arm & 5 (least deprived) & 0 & $(0)$ \\
& Targeted case finding & 6 & $(37.5)$ \\
& Routine care & 10 & $(62.5)$ \\
\hline
\end{tabular}

Note: IMD is a measure of socioeconomic deprivation based on postcodes. Abbreviation: IMD, Index of Multiple Deprivation. lifestyle behaviors (eg, exercise), and improved quality of life and disease prognosis by enabling earlier access to care. Some also felt that it would be cost saving for health services in the long term.

I guess the main advantage of screening is presumably to pick up the disease early so that ... I mean COPD is to some extent preventable and particularly if you treat it early and I guess the biggest advantage is ... you can encourage them to stop smoking ... if we treat it early, treat it effectively then hopefully there'll be fewer hospital admissions and therefore reducing the costs. [GP 8]

Several potential harms were also highlighted, including the impact on health services, such as increased workload, resources, and costs, as well as on patients, including the risk of overdiagnosis, the implications of diagnostic labeling on insurance costs, and creating anxiety.

... it's just that impact on workload really, whether primary care would just be overwhelmed if we started screening ... you could end up labelling people, which can have a huge impact, and they are fine. [Nurse 6]

\section{Diagnostic strategies}

Participants mainly reported that patients were investigated for COPD on an opportunistic basis when consulting the health services, particularly when presenting with suggestive symptoms. Others discussed using a more active approach such as screening at smoking cessation clinics. A wide range of factors were considered to be important triggers for considering COPD, such as smoking status and a history of asthma. Participants also highlighted the potential of clinical information systems to help identify and flag high-risk patients.

Also looking at computer data, we can set up searches on our computers, but it depends on how active people are at putting the information on the computer, then we can pull that information.... [Nurse 4]

Spirometry was described as essential for making a diagnosis of COPD, while screening tests such as handheld flow meters and respiratory questionnaires were discussed as potentially useful for assessing risk prior to diagnostic assessment. Some handheld flow meters were reported to feedback lung age, which was highlighted by several participants as being useful for promoting smoking cessation. Handheld flow meters were also described as quick and easy to use within a consultation. 
Table 3 Participant characteristics

\begin{tabular}{lllllllll}
\hline Characteristics & GP & \multicolumn{3}{c}{ Nurse } & \multicolumn{3}{c}{ Practice manager } & Total \\
\hline Number (\%) & 10 & $(50)$ & 7 & $(35)$ & 3 & $(I 5)$ & 20 & $(100)$ \\
Mean age in years (range) & 44.7 & $(3 I-73)$ & 46.7 & $(33-54)$ & 57.7 & $(55-6 I)$ & 47.4 & $(31-73)$ \\
Male (\%) & 7 & $(70)$ & 0 & $(0)$ & 0 & $(0)$ & 7 & $(35)$ \\
Mean years in practice (range) & 13.7 & $(2-35)$ & 15.7 & $(10-25)$ & 16.7 & $(I 2-25)$ & 14.9 & $(2-35)$ \\
\hline
\end{tabular}

Abbreviation: GP, general practitioner.

If someone has got appropriate symptoms, a smoking history, and a low $\mathrm{FEV}_{1}$ over V6, then we'll bring them in for formal spirometry ... overall I think it's not an unreasonable way to triage the people into proper spirometry. I think the key thing is not to make the diagnosis on the handheld stuff. [GP 7]

Most participants felt that the use of electronic risk prediction tools would be useful for identifying patients at high risk of undiagnosed COPD and even to help communicate risk to patients. Ease of use, provision of technical support, integration with existing clinical information systems, and the generation of automated prompts on electronic health records were seen as important factors for their implementation.

I think it's because we (nurses) always like something to refer to and we like to use tools, and I think sometimes that helps just to show the patient as well. Because we use a tool to assess cardiovascular risk ... I found it useful, because it illustrates to them for example if they're a smoker you can calculate their risk as a smoker, and then show them if you weren't a smoker it would be this ... So that's a visual thing for them to see. [Nurse 5]

A number of participants also highlighted the importance of being able to refer to secondary care, particularly for more challenging clinical presentations. One single-handed GP also commented on the need to refer patients to secondary care for medicolegal protection.

\section{Barriers to case finding for COPD}

Limitation of time, finances, and resources were seen as important barriers to implementing case finding and diagnosing COPD. Participants felt that primary care services were already stretched to capacity managing patients with established COPD and a lack of additional funding and resources would prohibit the implementation of case finding.

... just managing the patients who are already on the COPD register is a hell of an onerous task anyway so going out and case finding ... there's a cost implication, there's a man-time implication so unless it's well-resourced it's not going to happen. [GP 6]

There was also felt to be a significant lack of knowledge and expertise on COPD in primary care. This included poor understanding of spirometry; difficulties distinguishing between COPD, asthma, and COPD-asthma overlap disease; and underrecognition of the signs of COPD.

I suspect as a profession, we're not very good at picking up early signs of COPD either ... Partly because we, again, attribute a lot of their symptoms to their social habits smoking, lack of activity, environment. [GP 3]

Limited access to diagnostic services was also cited as a barrier, particularly in smaller practices, which often lack provision of in-house spirometry. Challenges to providing spirometry included costs of equipment and training, quality assurance, and availability of appropriately trained staff.

... you can't refer for spirometry, the only thing we could possibly do is buddy up with other practices, but not every practice has a practice nurse available to do spirometry or has a spirometry machine. [Practice Manager 1]

However, some participants did comment on the gradual improvement of diagnostic testing for COPD in the community.

It's getting better I think. I think there was a phase where people were just doing spirometry willy-nilly without necessarily having the right equipment, the training to use it properly. I think there has been a lot of improvement, particularly over the last couple of years with the accreditation.... [GP 3]

Several patient-related factors were also described as barriers to diagnosing COPD. These included poor attendance in primary care and late presentation with advanced disease. Patients were perceived to sometimes try to cope with symptoms for as long as possible without consulting the health services until suffering an acute exacerbation. Some felt that patients often underrecognized the significance of 
their symptoms or were not always forthcoming about them or their smoking habits.

... a lot of patients have symptoms but they just think that's what they should have because they're smokers so they don't often seek advice. [Nurse 7]

There was also a view that awareness of COPD among the general public was low, that patients were more likely to be aware of the more severe stages of the disease, and that smokers with undiagnosed COPD often have low expectations of their health. They also felt that communicating information about COPD was challenging.

If you said to the average man on the street, "What's COPD?" they wouldn't even know what it was ... when you do try to explain it to them, you get people going into panic mode then because it doesn't sound very nice ... there's just not enough educational publicity surrounding it. [Practice Manager 1]

Cultural barriers were also discussed, which present challenges to communicating risk as well as making a diagnosis because of underrecognition of exposures more common in the developing world, such as indoor air pollution from cooking fuels.

I think there is a linguistic barrier; increasing numbers of patients are from ethnic minorities and getting them up and looking at them, and actually understanding where their exposure has been .... You get all the little Asian ladies who cooked on open fires indoors and have COPD from that, but then they're not smokers ... So I think there's a lot of cultural things going on here. [GP 7]

\section{Facilitators for diagnosing COPD}

Training of health professionals was seen as one of the key facilitators for case finding and diagnosing COPD. Particular importance was attributed to spirometry training and acquiring a diploma in COPD, which several participating nurses had already achieved.

... two of our nurses are going to do a spirometry course to become more up-to-date and obviously qualified in doing spirometry, then we could offer more access to spirometry and possibly set up a breathing clinic .... [Nurse 4]

Access to community respiratory services, including specialist COPD nurses, and support from secondary care and community outreach were also seen as important, particularly since expertise on respiratory medicine in primary care was generally perceived to be low. Participants also discussed the importance of sharing diagnostic services between practices, which was especially important for smaller practices with limited service capacity.

... if say a patient was suspected with COPD and I've sent them off for spirometry, we normally send them off to a local service where they have this spirometry, and I guess luckily for us we do have a respiratory consultant reporting the spirometry findings as well which gives us recommendations. [GP 8]

The importance of educating patients and the public about COPD, including ethnic minority populations, was also discussed. This included communicating the symptoms of COPD, disseminating information at a community level, and use of social marketing and mass publicity.

I think more patient education, more information out there, more publicity ... I think it's got to be in the media really ... If you've got these sort of symptoms then see your GP, get it checked ... [Practice Manager 1]

\section{Perceptions of patients' responses to receiving a diagnosis of COPD}

Patients' responses to being diagnosed with COPD were perceived to be quite variable. Most participants felt that patients accepted their diagnosis and worked with their clinicians to improve their lifestyle behaviors, particularly in relation to smoking. Patients were perceived to sometimes even be relieved by the diagnosis, since this allows them to attribute a cause to their chronic symptoms.

I've not really had any genuine reluctance to accept a diagnosis ... I think they take on board what they have been told ... take on board the fact that by making lifestyle changes, they can significantly slow the progress of the process they have started. [GP 4]

However, it was acknowledged that patients were often shocked and upset by the diagnosis, particularly if they had family members who had severe disease, and also that there were implications for insurance costs and potentially employment. Some felt that patients were occasionally very reluctant to accept the diagnosis, particularly when they had no wish to give up smoking.

I think because it is a big shock, it is a big diagnosis, as I said it's got lots of implications with insurance. I think it frightens patients as well because they look at the worst case scenario and associations with oxygen ... [GP 2] 


\section{Discussion}

\section{Main findings}

Case finding for COPD is to some extent already occurring in primary care, and some health care providers believe that this will benefit patient care at the expense of applying high workload and cost pressures on the health service as well as risking overdiagnosis and creating anxiety among patients (Figure 1). Primary care providers are opportunistically diagnosing patients when presented with a suggestive clinical history, while others are keen to undertake active case finding using a range of approaches.

However, some important barriers to case finding were identified - limited service capacity, insufficient expertise on COPD and interpretation of spirometry, and restricted (but improving) access to diagnostic services. Perceived poor awareness of COPD and its symptoms among the public and the difficulty of communicating a diagnosis of COPD were also seen as barriers.

Investing in the training of health care professionals on COPD and spirometry; improving access to community respiratory services, including specialist $\mathrm{COPD} /$ respiratory nurses; and education campaigns to improve awareness of COPD in the general population were all suggested to improve the identification of patients with undiagnosed COPD.

Finally, health care professionals recognize that receiving a diagnosis of COPD can be an upsetting and life-changing event and patients' health beliefs and their response to the diagnosis can play an important role in subsequent management. Effective communication of the diagnosis is thus an important component of patient care.

\section{Relationship to other studies}

Like Walters et al, ${ }^{7,8}$ our study found that additional workload and resource requirements associated with case finding, as well as poor knowledge and confidence with spirometry interpretation, are likely to be barriers to diagnosing COPD in primary care. However, unlike their study, our participants did not express views of therapeutic nihilism. Instead, they largely felt that early intervention was likely to improve patient outcomes. Patients with COPD participating in a qualitative study in Sweden that explored their perspectives on receiving a diagnosis suggested that they would prefer the diagnosis to be given at an early stage. ${ }^{16}$ This also aligns with findings by Walters et $\mathrm{al}^{7}$ and the views expressed by health professionals in our study. A recent analysis of a large primary care database by Jones et al showed that opportunities to diagnose COPD in primary care are frequently missed. ${ }^{17}$ This was acknowledged by participants in our study, and a number of reasons for this were postulated, including health service and patient-related factors, such as underrecognition of symptoms.

The importance of support from secondary care was also highlighted by participants. In the Netherlands, an observational analysis of an asthma/COPD service to provide specialist support to GPs for diagnosing and managing patients with chronic respiratory diseases suggested that this support was feasible and effective in improving patient outcomes. ${ }^{18}$

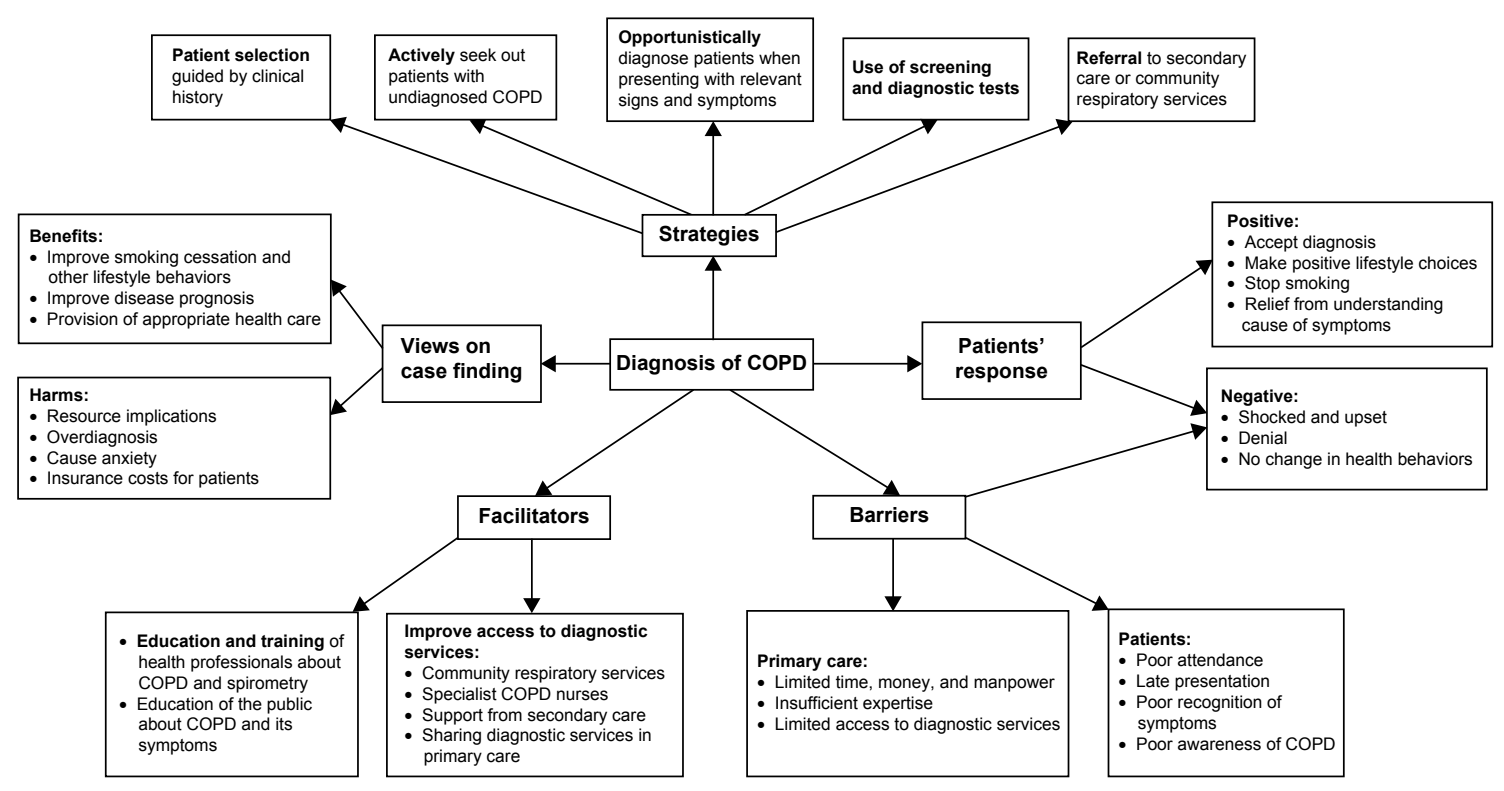

Figure I Summary of themes discussed by participants.

Abbreviation: COPD, chronic obstructive pulmonary disease. 
Interestingly, participants did not comment on the potential role of telemedicine for improving the diagnosis of COPD in primary care, which may be a potentially useful resource. For example, Bonavia et $\mathrm{al}^{19}$ in Italy demonstrated the feasibility and acceptability of telespirometry (where spirometry results were electronically transferred to pulmonary specialists and reports returned to primary care) among a large sample of GPs ( $\mathrm{n}=937)$ for diagnosing COPD.

\section{Strengths and limitations}

We sampled a variety of stakeholders to acquire a range of both clinical and nonclinical perspectives. Participants were from a number of practices with a wide range of characteristics, including those who had participated in both the case finding and routine care arms of the TargetCOPD trial. ${ }^{12}$

Patients were not interviewed as part of this study, so the views expressed were from a care provider perspective and may not necessarily reflect what patients personally experience. Transcripts were not returned to participants for validation of the themes, and the interpretation of the transcripts could have been influenced by the prior beliefs of the authors who are all involved in the evaluation of COPD case finding. Similarly, participating health care providers may possibly have been more engaged in the management of COPD than non-participants, and their views may have been biased toward proactive COPD diagnosis and treatment. The findings of this study should therefore be interpreted in this light.

\section{Implications for policy, practice, and research}

Improving the diagnosis of COPD in primary care will likely require investment in community respiratory services and training of health professionals on COPD and performance and interpretation of spirometry. Further research should explore public perceptions of COPD, including awareness of symptoms. Greater awareness may improve the likelihood that patients with undiagnosed disease access the appropriate services.

The benefits and harms of case finding highlighted in this study should be evaluated empirically in the long-term follow-up of case-finding trials. The findings of this study should also be compared to the views of patients, and the issues and implications surrounding the receipt of a diagnosis should be explored, addressing both the benefits and harms. The acceptability and feasibility of case-finding strategies should be qualitatively evaluated among care providers and patients alongside clinical trials evaluating their effectiveness.

\section{Conclusion}

The diagnosis of COPD in primary care may be improved by increasing access to community respiratory services and investing in the training of health professionals on COPD and spirometry. The benefits and harms of case finding should be empirically assessed in longitudinal studies to evaluate the overall effectiveness of detecting COPD early. Finally, the knowledge and attitudes of the public about COPD and its symptoms should be investigated to inform future education and awareness-raising initiatives and help those with undiagnosed disease access the appropriate care.

\section{Acknowledgments}

We would like to acknowledge all the participants who agreed to be interviewed, the transcribers Samuel Davies and Adrienne Dunn, the Bliss team for their support, and Joanne O'Beirne-Elliman for assisting with the ethics application. This paper presents independent research funded by the National Institute for Health Research (NIHR). The views expressed are those of the authors and not necessarily those of the NHS, the NIHR, or the Department of Health.

\section{Author contributions}

PA, REJ, and SH conceived the idea for this study. SH wrote the protocol with input from PA and REJ and applied for ethical approval. SH recruited the participants, conducted the interviews, and coded the transcripts. PA and REJ coded two transcripts to validate the initial coding framework. SH developed the themes that were discussed and verified by PA and REJ. SH wrote the paper with input from PA, REJ, and DAF. PA, REJ, and DAF are principal investigators of the TargetCOPD trial. All authors contributed toward data analysis, drafting, and critically revising the paper and agree to be accountable for all aspects of the work.

\section{Disclosure}

REJ, PA, and DAF are principal investigators of the TargetCOPD trial, which investigates the effectiveness and costeffectiveness of case finding for COPD in primary care. SH is conducting a $\mathrm{PhD}$ on case finding for $\mathrm{COPD}$ in primary care and is funded by an NIHR doctoral fellowship (DRF-201104-064). The authors report no other conflicts of interest in this work.

\section{References}

1. Lozano R, Naghavi M, Foreman K, et al. Global and regional mortality from 235 causes of death for 20 age groups in 1990 and 2010: a systematic analysis for the global burden of disease study 2010. Lancet 2012;380:2095-2128 
2. Gibson GJ, Loddenkemper R, Sibille Y, et al. Respiratory health and disease in Europe: the new European Lung White Book. Eur Respir J. 2013;42(3):559-563.

3. Buist AS, McBurnie MA, Vollmer WM, et al; BOLD Collaborative Research Group. International variation in the prevalence of COPD (The BOLD Study): a population-based prevalence study. Lancet. 2007;370:741-750.

4. Department of Health. An Outcomes Strategy for People with Chronic Obstructive Pulmonary Disease (COPD) and Asthma in England. London: Department of Health; 2011.

5. Dirven JA, Tange HJ, Muris JW, van Haaren KM, Vink G, van Schayck OC. Early detection of COPD in general practice: patient or practice managed? A randomised controlled trial of two strategies in different socioeconomic environments. Prim Care Respir J. 2013;22: 331-337.

6. Haroon S, Adab P, Griffin C, Jordan R. Case finding for chronic obstructive pulmonary disease in primary care: a pilot randomised controlled trial. Br J Gen Pract. 2013;63:26-27.

7. Walters JA, Hansen EC, Walters EH, Wood-Baker R. Under-diagnosis of chronic obstructive pulmonary disease: a qualitative study in primary care. Respir Med. 2008;102:738-743.

8. Walters JA, Hansen EC, Johns DP, Blizzard EL, Walters EH, WoodBaker R. A mixed methods study to compare models of spirometry delivery in primary care for patients at risk of COPD. Thorax. 2008;63: 408-414.

9. British Lung Foundation. Invisible Lives: Chronic Obstructive Pulmonary Disease (COPD) - Finding the Missing Millions. London: British Lung Foundation; 2007.

10. Global Initiative for Chronic Obstructive Lung Disease. Global Strategy for the Diagnosis, Management, and Prevention of Chronic Obstructive Pulmonary Disease. Marburg: Global Initiative for Chronic Obstructive Lung Disease; 2013.
11. Health and Social Care Information Centre. Quality and Outcomes Framework- 2011-2012, England Level: Clinical Domain, Chronic Obstructive Pulmonary Disease Data Tables. England: Health and Social Care Information Centre; 2013.

12. Jordan RE, Adab P, Jowett S, et al. TargetCOPD: a pragmatic randomised controlled trial of targeted case finding for COPD versus routine practice in primary care: protocol. BMC Pulm Med. 2014;14:157.

13. Mason M. Sample Size and Saturation in PhD Studies Using Qualitative Interviews. Oxford: Oxford Brookes University; 2010.

14. Morse JM. Determining sample size. Qual Health Res. 2000;10:3-5.

15. Gale NK, Heath G, Cameron E, Rashid S, Redwood S. Using the framework method for the analysis of qualitative data in multi-disciplinary health research. BMC Med Res Methodol. 2013;13:117.

16. Arne M, Emtner M, Janson S, Wilde-Larsson B. COPD patients perspectives at the time of diagnosis: a qualitative study. Prim Care Respir J. 2007; 16:215-221.

17. Jones RC, Price D, Ryan D, et al; Respiratory Effectiveness Group. Opportunities to diagnose chronic obstructive pulmonary disease in routine care in the UK: a retrospective study of a clinical cohort. Lancet Respir Med. 2014;2:267-276.

18. Metting EI, Riemersma RA, Kocks JH, Piersma-Wichers MG, Sanderman R, van der Molen T. Feasibility and effectiveness of an asthma/COPD service for primary care: a cross-sectional baseline description and longitudinal results. NPJPrim Care Respir Med. 2015;25: 14101.

19. Bonavia M, Averame G, Canonica W, et al. Feasibility and validation of telespirometry in general practice: the Italian "Alliance" study. Respir Med. 2009;103:1732-1737.
International Journal of COPD

\section{Publish your work in this journal}

The International Journal of COPD is an international, peer-reviewed journal of therapeutics and pharmacology focusing on concise rapid reporting of clinical studies and reviews in COPD. Special focus is given to the pathophysiological processes underlying the disease, intervention programs, patient focused education, and self management protocols.

\section{Dovepress}

This journal is indexed on PubMed Central, MedLine and CAS. The manuscript management system is completely online and includes a very quick and fair peer-review system, which is all easy to use. Visit http://www.dovepress.com/testimonials.php to read real quotes from published authors. 